\title{
AL REVÉS. APUNTES SOBRE EL DEVENIR NIETZSCHE-ARTAUD
}

Sara Baranzoni

En la tentativa de traer a la luz otras formas de devenir, más allá de aquellas potentemente descritas por Deleuze y Guattari, propongo en este ensayo un breve recorrido filosófico alrededor del pliegue estético de un problema biológico y metafísico que atormenta a los dos grandes pensadores que se han hecho protagonistas de una encrucijada radical para los tiempos por venir: Friedrich Nietzsche y Antonin Artaud.

Antes de empezar, es necesario especificar que no se tratará de un ejercicio de comparación general entre los dos autores, sino de trazar una serie de líneas abiertas, o rasgos de devenir, incrustadas sobre una tendencia al revés. Esto porqué, como es bien conocido, ambos son protagonistas de varias tentativas de reversar, por ejemplo, el platonismo, la religión, el pensamiento occidental y los puntos de vista disciplinares - la metafísica y la racionalidad moderna, el teatro y la escritura -, el papel del cuerpo y de sus funciones, así como aquello del filosofo-escritor, para, de alguna forma, rehacer la vida. He aquí un primer elemento que los une: y si en este sentido no existen evidencias contundentes de que Artaud se haya inspirado o no en Nietzsche, si y cuanto lo haya leído (aunque es cierto que en su Van Gogh lo mencione entre los varios "suicidados de la sociedad", o en otros textos cite, por ejemplo, desde Ecce Homo), veremos que no es el propósito filológico lo que nos anima. En otras palabras, lo que importa no es establecer si entre los dos cabría alguna continuidad o discontinuidad, si se pueda hallar o no un parentesco o una hermandad. Más bien, propongo pensar una posible relación bajo la categoría del doble, según la introduce JeanPierre Vernant en su obra Mito y pensamiento en la Grecia Antigua (1973).

La figura del doble no se convoca aquí casualmente. Para ambos pensadores se trata de un tropo recurrente y de, algunas maneras, ligado al teatro. Si, según Nietzsche, el doble se refiere al espíritu trágico y pre-trágico en el cual el teatro debería buscar inspiración para hacer aparecer en el mundo fenoménico una realidad que redobla la vida, creando algo incluso superior - así que, al final, es la realidad efectiva la que imita las figuras del arte (NIETZSCHE, 2007, §24), para Artaud, el pensamiento del doble es el corazón del "teatro de la crueldad", expresando la necesidad de crear un puente entre la vida y lo real (ARTAUD, 1978). Algo que, nietzscheanamente, como sugiere Camille Dumoulié (1992), podríamos también llamar "voluntad de poder": términos que expresan la lógica de la vida - una lógica nueva, una ética más bien, que como veremos, ambos autores levantan como alternativa a las leyes de la racionalidad y de su moral.

El doble, pues, no sería mistificación o superación de lo real, sino su intensificación aunque en planos distintos; y es así precisamente como Vernant lo presenta para mostrar cómo los griegos condensaron en una forma-idea (eidos) una serie de «poderes del más allá que pertenecen al dominio de lo invisible» (VERNANT, 1973, p. 302).

Según relata Vernant, en el pensamiento griego la condición humana se caracteriza por su aspecto doble y ambivalente que se refleja en una serie de parejas - masculino y femenino, vida y muerte, soma y psique - y cuya relación no se reduce a una entre contrarios, opuestos, sino que introduce una verdadera "categoría psicológica", extremadamente compleja si no se abandona la organización mental típica de la lógica occidental. Pensemos, por ejemplo, sugiere Vernant, en los ritos funerarios y, concretamente, en la figura del colossos como substituto simulacro - del cadáver ausente: el colossos no es simplemente "la imagen" del muerto que fija sus rasgos en la piedra, no es su imitación, su fotografía, su "eterna repetición de lo mismo". Más bien, es su vida otra, su vida "más allá", su doble, como el mismo muerto es un doble del vivo, lo mismo, pero, como en Nietzsche, en otra capa de lo real. Asimismo, en el caso de los desaparecidos de los cuales no se ha podido hallar y homenajear sus cuerpos y, por ende, «el 
difunto - o más bien su "doble", su psyqué - permanece errante sin fin entre el mundo de los vivos y el de los muertos» (VERNANT, 1973, p. 304), el colossos puede constituir una solución farmacológica ${ }^{1}$ : redoblar el doble (el muerto), así restituyéndole así su vida y su presencia.

Lo importante es recalcar aquí que un doble no es una copia que está relegada en a otro mundo, sino la cualidad de este ser que puede permitir establecer un contacto entre mundos, abriendo así otro nivel de presencia, una presencia «insólita y ambigua que es también el signo de una ausencia» (VERNANT, 1973, p. 305). Porque, tal y como este lo explica:

Un doble es algo completamente distinto a una imagen. No es un objeto "natural", pero tampoco es un producto mental: ni una imitación de un objeto real, ni una ilusión del espíritu, ni una creación del pensamiento. El doble es una realidad exterior al sujeto pero que, en su misma apariencia, se opone por su carácter insólito a los objetos familiares, al decorado ordinario de la vida. Se mueve en dos planos que muestran al mismo tiempo una notable disconformidad: en el momento en que se hace presente se descubre como no siendo de aquí, como perteneciente a otro lugar inaccesible. (VERNANT, 1973, p. 307)

Así, un ejercito de dobles se despliega: un viviente, su doble, y el doble del doble, cada uno siendo multiplicación de lo mismo, y por esto capaz no sólo de comunicarse con sus otras partes a través de los umbrales de real que atraviesa, sino también de modificar los acontecimientos relativos a ellas y, por supuesto, los efectos.

Es precisamente bajo esta luz que queremos configurar la relación Nietzsche-Artaud, como una entre autores que se mueven en planos disconformes, y que, en cierto sentido, se pertenecen y se intercambian de papel, de cara, de sentido, en una superposición de planos que no es identificación ni transformación, sino devenir, según la celebre formula de Deleuze y Guattari:

Un devenir no es una correspondencia de relaciones. Pero tampoco es una semejanza, una imitación y, en ultima instancia, una identificación. [...] Devenir no es progresar ni regresar según una serie. $Y$, sobre todo, devenir no se produce en la imaginación, incluso cuando ésta alcanza el nivel cósmico o dinámico [...]. Los devenires animales no son sueños ni fantasmas. Son perfectamente reales. Pero, ¿de qué realidad se trata? (DELEUZE Y GUATTARI, 2002, P. 244)

Para decirlo de forma clara: lo que queremos demostrar no es que Nietzsche deviene de algunas maneras Artaud o viceversa. El devenir es más bien el tipo de línea que los une, una línea que no prevé un punto de origen y uno de destino, sino una alianza molecular en los dos sentidos contemporáneamente. «Devenir no es ciertamente imitar, ni identificarse» (DELEUZE Y GUATTARI, 2002, P. 245), más bien, es crear un mundo, otro mundo, varios mundos: hacer el doble, y así constituir un sistema de intercambio entre planos distintos.

Ahora bien, si ya hemos mencionado las distintas capas que cada uno de los dobles habitaría, así como los intercambios recíprocos que pueden verificarse, proponemos ver el doble en acción como una figura singular del derrocamiento (del "al revés"), con el fin de resaltar otro carácter, uno de los rasgos de devenir que lo atraviesa: la disolución del yo, no como división o reducción, sino por multiplicación, propagación, contagio, hasta la identidad infinita, paradoja del puro devenir (DELEUZE, 2005, p. 28) y a la perdida del nombre propio.

\footnotetext{
${ }^{1}$ La farmacología, referida al termine griego pharmakon, remedio y veneno al mismo tiempo, es otro de los aspectos emblemáticos que enmarcan en una continua ambivalencia la cultura griega, según la cual, muy a menudo, el antídoto se oculta en el mismo problema (veneno) y viceversa.
} 
En fin, insertando Nietzsche y Artaud en una relación de este tipo - el doble - hay necesariamente algo más que se encuentra también en el concepto deleuziano de devenir: la idea de multiplicación, hasta disolución, del yo. Este será el rasgo principal de devenir que convocaremos aquí.

\section{Al revés}

Uno de los elementos que caracterizan este devenir Nietzsche-Artaud y que nos permite pensarlo en términos de doble es el del pensamiento de "al revés" o el de "a la inversa": ambos transitando por un cierto deseo de rehacer la vida, sobre el cual han escrito - y se han escrito cientos de páginas, pero en relación con el cual quisiera referirme a dos fragmentos en particular. El primero, muy conocido, es el final de Para terminar con el juicio de dios, donde introduce la cuestión del cuerpo sin órganos:

El hombre está enfermo porque está mal construido.

Átenme si quieren, pero tenemos que desnudar al hombre para rasparle ese microbio que lo pica mortalmente dios y con dios sus órganos porque no hay nada más inútil que un órgano. Cuando ustedes le hayan hecho un cuerpo sin órganos lo habrán liberado de todos sus automatismos y lo habrán devuelto a su verdadera libertad. Entonces podrán enseñarle a danzar al revés como en el delirio de los bailes populares y ese revés será su verdadero lugar.

(ARTAUD, 1975, p. 30-31, cursiva mía)

El segundo es de Nietzsche, tomado de los Fragmentos póstumos 1888:

Contramovimiento

Anti-Darwin.

Lo que más me sorprende al revisar los grandes destinos del ser humano es ver siempre ante mis ojos lo contrario de lo que hoy día Darwin y toda su escuela ven o quieren ver: la selección a favor de los más fuertes, de los mejor dotados, el progreso de la especie. Con las manos se toca justamente lo contrario: la supresión de los casos afortunados, la inutilidad de los tipos más altamente logrados, el inevitable dominio de los tipos mediocres, e incluso de los que están por debajo de la media. Mientras no se nos indique la razón de por qué el ser humano es la excepción entre las criaturas, me inclinaré a prejuzgar que la escuela de Darwin se ha equivocado en todas las cuestiones. Esa voluntad de poder en la que yo vuelvo a reconocer la razón y el carácter últimos de toda alteración nos proporciona el medio de saber por qué precisamente la selección no se lleva a cabo en favor de las excepciones y de los casos afortunados: los más fuertes y los más felices son débiles cuando 
tienen en su contra los instintos de rebaño organizados, la pusilanimidad de los débiles, la superioridad numérica. Mi visión global del mundo de los valores muestra que, en los valores supremos que hoy día están dispuestos por encima de la humanidad, no predominan los casos afortunados, los tipos seleccionados: al contrario, los tipos de la décadence — quizá no haya nada más interesante en el mundo que este espectáculo indeseado...

Por extraño que suene: se ha de armar siempre a los fuertes contra los débiles; a los felices contra los desgraciados; a los sanos contra los depravados y los lastrados con taras hereditarias. Si se quiere una fórmula de la realidad que constituya una moral: esta moral dice así: los mediocres tienen más valor que las excepciones, los productos de la decadencia más que los mediocres, la voluntad de nada predomina sobre la voluntad de vida [...]

Yo veo a todos los filósofos, veo a la ciencia de rodillas ante la realidad de una lucha por la existencia inversa a la lucha que enseña la escuela de Darwin - es decir, por todas partes veo que predominan, que sobreviven, aquellos que comprometen la vida, el valor de la vida. - El error de la escuela de Darwin se ha convertido para mí en un problema: [...] Que las especies representen un progreso es la afirmación más irrazonable del mundo: de momento, las especies representan un nivel, -

que los organismos superiores se hayan desarrollado a partir de los inferiores, hasta ahora ningún caso lo ha demostrado -

yo veo que los inferiores tienen preponderancia por la cantidad, por la habilidad, por la astucia $[\ldots]$

- yo encuentro la «crueldad de la naturaleza», de la que tanto se habla, en otro lugar: la naturaleza es cruel con sus hijos afortunados, conserva y protege y ama a les humbles [humildes] — exactamente como----------

$* * *$

In summa: el crecimiento del poder de una especie está quizá menos garantizado por la preponderancia de sus hijos afortunados, de sus individuos fuertes, que por la preponderancia de los tipos mediocres e inferiores... En estos últimos está la gran fertilidad, la duración; con los primeros crece el peligro, la devastación súbita, la rápida disminución de número. (NIETZSCHE, 2008, 14[123], p. 561-562])

Mucho se ha dicho de la enigmática expresión "cuerpo sin órganos", así como de la furia manifestada por Artaud en contra de la anatomía establecida y de la mala construcción del cuerpo. Como lo afirma también en Artaud el Momo (1989, p. 59 y siguientes), según Artaud, las necesidades anatómicas de la medicina moderna han hecho del cuerpo humano lo que hoy llamaríamos una serie de datos ordenados racionalmente para obedecer a necesidades representativas disciplinares. Reducir el cuerpo a este tipo de estructura (un soporte, mejor, el subjetil $^{2}$ ) a la cual se enganchan elementos fijos que funcionan precisamente confirmando el orden ya establecido, no tiene nada que ver con la "verdadera anatomía" que él anhela. Sin embargo, la de la medicina solo es una de las formas que la transcendencia (Dios) toma a lo largo del (des)pensamiento del poeta francés; una transcendencia que nos fuerza a reconocernos en ella, a atribuirnos identificaciones estables haciéndonos perder o dejando inutilizadas todas aquellas posibilidades que "no sirven" para sus fines y que el cuerpo podría, en cambio, atravesar.

Pero antes de seguir con este punto, volvamos a Nietzsche, dejando que Artaud permanezca un poco en la sombra, como un eco que, tal vez, se podrá escuchar a través de su doble.

\footnotetext{
${ }^{2}$ Como recuerda Jacques Derrida (2011), en sus obras, Artaud utiliza esta palabra antigua, probablemente derivada del italiano, tres veces, y para hablar de sus dibujos. Soporte, materia y sujeto, sujeto sujetado y al mismo tiempo lanzado en el mundo, que como tal debe ser forcenado y custodiado para que pueda producir una opera.
} 


\section{Multiplicación del yo}

Como es bien conocido, Nietzsche es el agente de un derrocamiento total de la filosofía moderna, y para poder alcanzar este objetivo deberá empezar por un punto de partida totalmente opuesto. De hecho, si Descartes partía del alma, afirmando (así es el título de la segunda meditación) «la naturaleza del espíritu humano [...] es más fácil de conocer que el cuerpo» (DESCARTES, 2011), Nietzsche partirá entonces del cuerpo con el mismo argumento en forma de imperativo: «El fenómeno del cuerpo es el fenómeno más rico, más claro, más aprehensible: anteponerlo metódicamente, sin decidir nada sobre su significado último». (NIETZSCHE, 2008, 5[56], p. 161).

Sería muy ingenuo pensar que poner al cuerpo en lugar del alma es suficiente para acabar con la racionalidad moderna. No es suficiente oponer al mundo de los objetos y de los cálculos un mundo de pulsiones y afectos para vencerlo. Asimismo, afirmar de forma sencilla que "el cuerpo piensa" no sería nada más que cambiarle de nombre al sujeto sin derrocar su posición prioritaria como motor del pensamiento. Realmente, hasta cuando la filosofía se quedará arraigada a la cuestión del sujeto dado en tanto unidad - sea esta unidad fundada en el espíritu o razón o en el cuerpo - y aspirará a la "representación exacta" del sujeto por él mismo, la lógica del pensamiento seguirá siendo cartesiana.

¿Dónde se sitúa entonces el destronamiento de la razón occidental, este movimiento al revés del pensamiento que propone Nietzsche? Si prestamos atención al fragmento citado, podemos vislumbrar una línea de acción fundamental ligada al tema de la biología y de su bios - la vida. Lo que distingue los seres vivientes en la filosofía de Nietzsche es la posibilidad de nutrirse, y si pensamos en las funciones peculiares del cuerpo vivo, esto, en cierto sentido, vale también: entre ellas, la de acumular experiencias de las cuales el cuerpo se nutre y digiere es vital. La tendencia general de los órganos es la de "ingerir" los elementos externos y la de asimilarlos, es decir, volverlos idénticos (ad-similare) a lo que ya conoce o ha probado, convirtiendo así toda novedad en "lo mismo". Si así no fuese, cada elemento novedoso se volvería en un correspondiente riesgo de destrucción (envenenamiento) del organismo; en cambio, si asimilada, la novedad se torna en parte orgánica respeto al sujeto unitario, el in-dividuo. En este sentido, el cuerpo no se aleja demasiado de la razón cartesiana, que define el yo como aquel que juzga, que quiere y siente lo mismo en todo lo que experimenta, así organizando el mundo en objetos calculables (representaciones) de los cuales se puede apropiar reconduciendo todo al idéntico ${ }^{3}$.

¿Y si el cuerpo fuera algo más? De hecho, como afirma la filósofa francesa Barbara Stiegler (2010), Nietzsche se apresura en afirmar que el cuerpo no constituye un ego, ni un $i d$, («Los continuos tránsitos no permiten hablar de "individuo", etc.; el "número" mismo de los seres es fluctuante», NIETZSCHE 2010, 36[23], p. 802), sino algo que siente y se experimenta como varios: «Lo más asombroso es [...] el cuerpo: no puede uno dejar de admirarse de que haya sido posible el cuerpo humano: cómo la unión de tantos y tantos seres vivos, cada uno dependiente y sometido y, sin embargo, en cierto sentido, a su vez, mandando y actuando con voluntad propia, pueda vivir, crecer y subsistir durante un tiempo como un todo [...]!» (NIETZSCHE 2010, 37[4], p. 812). Según Nietzsche, la más minúscula célula es ya un organismo, una pluralidad de órganos articulados entre ellos, una colectividad, un nosotros compuesto de consciencias y voluntades. Así, en el cuerpo lo que hay que resaltar es precisamente su riqueza, su complejidad, su "prodigiosa diversidad". Al mismo tiempo, la acción del cuerpo no sería una simple asimilación, celular o lógica, que convierte todo en lo mismo, sino la batalla para la superación de una serie de obstáculos, lo que genera un sentimiento de poder. Este es el primer punto que distingue su filosofía de las modernas: el cuerpo vive en una búsqueda deliberada de todo lo que resiste a la digestión, con un cierto gusto por lo

\footnotetext{
${ }^{3}$ Se trata, de hecho, de la misma crítica al juego de la identidad que Deleuze propone en Diferencia y Repetición (2002).
} 
problemático, o por lo que, en los fenómenos, excede lo que podemos comprender de ellos. Cada nuevo conocimiento, cada nueva experiencia, es de por sí dañina, y el cuerpo, alimentándose, es forzado a confrontarse con una alteración pasiva de su equilibrio. Y si de alguna forma intenta hacer soportable la novedad, reconociéndola y absorbiéndola, por otro lado, siempre queda una parte que permanece radicalmente otra. Es precisamente dicha parte que, constituyendo una reserva de resistencia a la asimilación, deviene el sustrato capaz de generar variaciones $\mathrm{y}$, por consiguiente, nuevas interpretaciones. Además, todo esto pasa por el dolor o, más general, por los afectos: «comprender es, en su origen, una sensación de sufrimiento, y reconocimiento de un poder extraño» (NIETZSCHE 2010, 7[173], p. 211), que genera heridas que afectan el sujeto viviente en su profundidad, y de las cuales él trae su poder de afirmación.

Si utilizamos esta lógica, que está en la base de una verdadera inversión en el pensamiento, claramente podemos comprender el aforismo precedente: el cuerpo aparece inmediatamente como la evidencia más deslumbrante de la diversidad que desafía los procesos asimiladores establecidos. Pensada en este sentido, la asimilación, es decir, la reducción a uno, adquiere un significado completamente distinto: más que para limitar los riesgos, ella apuntaría a una mayor apertura del viviente en relación con lo que ocurre, ya que es precisamente gracias a lo que lo afecta que el sujeto incrementa su poder.

Todo esto inaugura, además, otra cuestión ligada al cuerpo: la identidad. Viendo la unidad caracterizada por la complejidad debida a la multiplicidad de las perspectivas que los órganos generan y a su carácter procesual, incumplido, para Nietzsche el ego siempre se deberá entender como proyecto, como ficción o sueño del sujeto vivo: una "prodigiosa reunión de lo diverso" que actúa solo en la medida en que padece, que no se reconstruye o inventa sino a partir de sus mismas heridas. De por sí, entonces, «el concepto de "individuo" es falso. Estos seres aislados no existen en absoluto: el centro de gravedad es algo mutable; la continua producción de células, etc. provoca un continuo cambio del número de estos seres» (NIETZSCHE 2010, 34[123], p. 742). Y si el centro de gravedad del cuerpo es algo mutable, se puede entonces deducir que no existe necesidad alguna en la formación de un organismo, así como de la consciencia que, presumiblemente, debería comandarlo. Es más, ella es «el último y más tardío desarrollo de lo orgánico y, por consiguiente, lo menos acabado y menos fuerte en él» (NIETZSCHE, 1990, 11, p. 35). Superflua, extremadamente imperfecta y desacertada, según Nietzsche, la consciencia aparece, además, solo cuando la totalidad quiere subordinarse a una totalidad superior. La fe en el todo como organismo, pues, es también para Nietzsche solo la nueva versión de la fe en dios, instituida por una ciencia que funciona al revés dado que nos proporciona como esencial, universal y eterno algo que, más bien, se ha generado por error. Es en este sentido que podemos comprender la afirmación de que el origen de la vida no sería, como lo pensó Darwin, la mejor adaptación posible a las condiciones reales encontradas, sino su máxima falsificación: «es, entonces, probable que lo que originariamente engendró la vida, fuera precisamente el más grosero de los errores que se pueda pensar», y que, asimismo, la vida «se sigue engendrando por medio de errores» (NIETZSCHE 2008b, 11[320], p. 829).

\section{La ficción del yo y la selección artificial}

Según Nietzsche, la función principal de este sujeto ficcional, vivo y activo es la selección: el viviente pasa su tiempo tratando de reponerse activamente de lo que lo afecta - de su sufrimiento - para lo cual la síntesis o asimilación no sería más que un caso particular. El viviente es llamado a cuidarse, pero no en sentido catártico: la lucha en contra de los daños que la novedad produce no se lleva a cabo con el fin de purgarse, de liberarse o de limpiar el espíritu en vista de una "paz" por venir. Más bien, ella es parte de la comprensión y aceptación de la 
crueldad originaria de la vida, en relación con la cual el sujeto se forma como tal, no negándola o intentando eliminarla, sino eligiéndola, es decir, afirmándola constantemente. Pero, si el sujeto es ficción, si está más bien compuesto por una pluralidad de "voluntades de poder", ¿cómo se actuará esta elección? ¿cómo se homogeneizarán o armonizarán las distintas partes, ya que en la guía de este proceso no está un yo (o una conciencia) planificador(a) o transcendental? Lo único que ayuda en esto, sostiene Nietzsche, es la memoria, en tanto capacidad de retener en sí la huella del otro, de soportarlo, reconociéndolo como mismo y arriesgándose a acumular una serie de resistencias a este mismo que, como decíamos, devienen potencial de ruptura para una nueva interpretación de lo que adviene.

Esta es la lucha por la existencia al revés de la que habla Nietzsche. Como en cada lucha, el viviente siempre tiene que adoptar el criterio adecuado frente a lo que le afecta, frente a la potencia deflagradora del acontecer. Pero, si no hay sujeto ni categorías transcendentales à la Kant, ¿cuál será entonces este criterio y a qué se adecuaría? Encontramos aquí la gran novedad de Nietzsche, o lo que podríamos pensar como la inversión de Darwin: no sería la conservación de sí (ya que el "sí" no existe) la que guiaría esta selección, sino la misma voluntad de poder, el impulso de ir más allá de cualquier "sí", exceso de voluntad y de vida que puede siquiera incluir a la muerte como posibilidad de conservación de la vida en general (y no de "una vida" particular). «A fuerza de morir, acabé ganando la inmortalidad real», diría Artaud. De todas maneras, no se trata de un proceso de perfeccionamiento o mejoramiento - de la "selección natural de lo más fuerte", como pensaba Darwin - sino de una "selección artificial", basada en lo que Georges Canguilhem (1971) llamará la "normatividad de la vida", una vida activamente cruel y peligrosa, de la cual el viviente debe devenir a la altura con todos los medios de los que dispone. He aquí un ulterior volcamiento de valores: lo útil para la vida no será lo que la hace más estable, lo que asegura la continuidad del individuo, sino lo que la desestabiliza, desencadenando fuerzas de reparación capaces de desplegar al máximo el poder de lo nuevo (STIEGLER 2010, p. 110). La tentativa de pensar una articulación posible entre el padecer y el actuar se traduce en activación de la potencia de reparación de la vida, en la "gran salud" que expresa el poder de la vida, su potencia falsificadora. De hecho, es así que el humano devino tal: luchando contra las heridas, redoblando los procesos fisiológicos de reparación con la invención de nuevas formas que hacen que nunca pueda coincidir consigo mismo. Además, es precisamente en el choque entre fuerzas - activas y reactivas - que podemos entender también la paradójica necesidad de «armar los fuertes contra los débiles»: los fuertes, atravesados por fuerzas activas, enfrentan la crueldad y la asumen ingiriendo toda posible novedad posible, hasta poner en riesgo su misma existencia. Con menos posibilidades de sobrevivir, ellos devienen entonces la excepción, mientras que los débiles, los asimiladores, los identitarios, proliferan ${ }^{4}$. Por esta razón la vida es crueldad: porque está lista para eliminar sus mejores productos, para poder afirmarse como producción de novedades. O, si queremos decirlo con las palabras de Artaud: la crueldad es el rigor de una elección, una decisión implacable y la aplicación en su perseguimiento, determinación irreversible, absoluta (ARTAUD 1978). La lógica de la crueldad, entendida como principio ético, hace de la vida la experiencia más peligrosa para el ser humano, hasta superar así también el límite último que el orden del pensamiento occidental puede aceptar: la garantía de la existencia misma del hombre.

\section{Artaud, el cuerpo y la danza al revés}

Notoriamente, todo el trabajo de Antonin Artaud, desde sus proyectos teatrales hasta sus dibujos, escritos, viajes, conferencias, para terminar con su obra maestra que hubiera debido ser el

\footnotetext{
${ }^{4}$ Se trata de la misma interpretación proporcionada por Deleuze (1998).
} 
símbolo de su regreso a la normalidad y una excepcional emisión radiofónica - Para terminar con el juicio de dios (1975) -, se basa en el trabajo sobre el cuerpo. En principio, en los tiempos del teatro de la crueldad, Artaud intenta rehacer el cuerpo, no solamente con su entrenamiento vocalfísico basado en la respiración, sino, además, con gestos y escritura. Hay que entender también que para él el cuerpo no es solamente el del ser humano, sino todo elemento que está compuesto por partes organizadas y, por ende, expropiadas. El cuerpo, robado por un "dios" que ha nacido «haciéndose pasar por mí mismo», solo puede volver a nacer a través del teatro: «acto peligroso y terrible», «crisol de fuego y carne». El teatro es la sede de una verdadera transformación anatómica (ARTAUD, 1975b, p. 81). Y también cuando Artaud “deja" el teatro de los espectáculos para operar una "nueva génesis" directamente sobre su cuerpo, esta podrá considerarse, nietscheanamente, como el despliegue de las multíplices fuerzas que el cuerpo guarda en sí, que acontece solo y cuando el cuerpo puede deshacerse de su identidad. Una vez que haya vuelto a cambiar, el cuerpo devendrá inmortal, ya que «la muerte es un estado inventado [...] El cuerpo humano sólo muere porque se ha olvidado transformarlo y cambiarlo» (ARTAUD, 1975b, p. 81-82). Pero, ¿por qué el cuerpo no cambia? Precisamente porque se ha hecho creer a los seres humanos que solo importa la razón, la conciencia, o el espíritu, ya que ellos son inmortales. En cambio, Artaud redobla a su manera el gesto anti moderno de Nietzsche afirmando que

El mental no hace al cuerpo, pero el cuerpo produce lo mental; y el cuerpo de todos libera un espíritu aterrador porque hace 4000 años que el hombre tiene una anatomía que ha dejado de corresponder a su naturaleza. La anatomía en la que estamos agrupados es una anatomía creada por burros licenciados, médicos y científicos que nunca han podido entender un cuerpo simple y que necesitaban para vivir encontrarse en un cuerpo que les respondiera y que ellos entendieran. Y ellos tomaron el cuerpo humano y lo rehicieron de acuerdo con los principios de una lógica clara y sólida,

punto por punto,

órgano por órgano,

analítica a su manera.]

(ARTAUD, 2004, “Ainsi donc, à vous bien comprendre...” [1947], p. 1188)

La razón no es que un órgano auxiliar, diría Nietzsche, y nuestros órganos están organizados sobre el error. En otros textos del mismo período, Artaud se refiere a un tiempo en que semejante funcionalización del cuerpo todavía no existía, o no había sido impuesta, un tiempo en que el cuerpo era voluntad pura, en que «el hombre era un árbol sin órganos ni función», pero «con voluntad que camina», afirmando enseguida que la gran mentira fue «hacer del hombre un organismo | ingestión, asimilación, | incubación, excreción, [...] este árbol humano que avanza esto era: | una voluntad que decide de sí en cada instante, | sin funciones ocultas, subyacentes reguladas por el inconsciente» (ARTAUD, 2004, "Lettre à Pierre Loeb" [1947], p. 1602-1603).

El teatro de la crueldad deviene así el lugar especifico donde no se busca volver a un estado originario perdido, sino reactivar las fuerzas vitales, la voluntad, a su enésima potencia, hasta hacer «aflorar la vida bajo la piel como el picor de una terrible irritación» (ARTAUD, 2004, "Lettre à Maurice Saillet" [1947], p. 1204-1205), y donde ya no se teme la muerte. No es necesario insistir mucho para llegar a pensar semejante teatro como una "generación al revés" (DERRIDA, 2011) donde el cuerpo, una vez recortado de su soporte orgánico y una vez superados los terribles sufrimientos necesarios para esto, conocerá «el regreso de una eterna salud» (ARTAUD, 2004, "Le théâtre de la cruauté" [1947], p. 1657). Asimismo, y una vez más, la crueldad de la vida (y para volver a la vida), no será para pensarla como una personificación de la naturaleza ("la naturaleza es cruel"), sino, más bien, como una fuerza que se agita en la naturaleza, que dicta su ética, "mas allá 
del bien y del mal". Esto no quiere decir excluyendo el bien o el mal hacia un antes ("la inocencia perdida") o un después (un futuro mítico que los excluya) de ellos, sino precisamente "por sus mismos medios", en la misma medida en que Artaud pudo ir más allá del teatro, transcendiéndolo con sus mismos medios y llevándolo así a sus extremas consecuencias.

Para concluir, pues, ¿qué será entonces esta danza al revés de la que habla Artaud, o que, más bien, el doble Nietzsche-Artaud opera? Una danza de los órganos, de las células, de la biología, por supuesto, pero con ella, la necesidad de un cuerpo que destruye su organización racional para encontrar su propia razón: "la gran razón" del cuerpo (Nietzsche), no un principio directivo, sino una pluralidad a sentido único, una guerra y una paz, donde no existe un centro de poder inmutable, ningún jefe preciso que asegura su dirección, sino que es el impulso que se afirma en un momento dado a tomar la cabeza de este organismo. Este tipo de organismo donde los agenciamientos entre órganos no son más que tentativas aleatorias, errores indispensables para la vida, es un medio para de aumentar el conflicto y, con ello, el grado de potencia del cuerpo mismo.

Entre los paridos son muchos los inútiles, los que respecto de la meta de la especie son frágiles e ineficaces - pero superiores: jen esto tenemos que fijarnos! [...] La meta, obviamente, es que el hombre llegue a ser tan regular y estable como ha sucedido con la mayoría de las especies animales: se han adaptado a las condiciones de la tierra, y demás, y en esencia ya no cambian. El hombre todavía cambia - está haciéndose. (NIETZSCHE, 2008b, 11[44], p. 768)

Es así que el cuerpo deviene el campo de batalla de los órganos, lugar de encuentros y desafíos que desencadena miembros y rasgos "percibidos como abyectos" (Artaud) en la danza cruel de la asimilación y de la novedad, de la crueldad y de la voluntad, desde la cual emana el vapor del acontecimiento, doble de lo real sin ser menos real: el acontecimiento del doble mismo, que, entre las chispas de la identidad (des)hecha en mil pedazos, emana como tal, acompañado por un ejército de otros dobles, múltiples. Eterno diálogo, aquello imaginario entre sí del doble Nietzsche-Artaud, el diálogo inolvidable de la multiplicidad.

$\mathrm{N}$ : Todavía hay otro punto en el que, una vez más, yo soy meramente mi padre... A: Yo, Antonin Artaud soy mi hijo, mi padre, mi madre y yo.

$\mathrm{N}$ : Yo soy todos los nombres de la historia...

A: ¿Quién soy?

¿De dónde vengo?

Yo soy Antonin Artaud

y apenas yo lo diga como sé decirlo inmediatamente

verán mi cuerpo actual estallar

y recogerse

bajo diez mil aspectos notorios un cuerpo nuevo

en el que ustedes no podrán

nunca jamás

olvidarme.

\section{Referencias}

ARTAUD, A. Para terminar con el juicio de dios y otros poemas. Traducción de María Irene Bordaberry y Adolfo Vargas. Buenos Aires: Caldén, 1975.

ARTAUD, A. "El teatro y la ciencia", en Para terminar con el juicio de dios y otros poemas. Traducción de María Irene Bordaberry y Adolfo Vargas. Buenos Aires: Caldén, 1975b, p. 80-85. 
ARTAUD, A. El teatro y su doble. Traducción de Enrique Alonso y Francisco Abelenda. Barcelona: Edhasa, 1978.

ARTAUD, A. Artaud el Momo. Traducción de Sara Irwin. Buenos Aires: Need, 1998.

ARTAUD, A. Euvres. Editado por Evélyne Grossman. Paris: Gallimard, 2004.

CANGUILHEM, G. Lo normal y lo patológico. Traducción de Ricardo Postchart. Buenos Aires: Siglo XXI, 1971.

DELEUZE, G. Nietzsche y la filosofía. Traducción de Carmen Artal. Barcelona: Anagrama, 1998.

DELEUZE, G. Differencia y repetición. Traducción de María Silvia Delpy y Hugo Beccacece. Buenos Aires: Amorrutu, 2002.

DELEUZE, G. Lógica del sentido. Traducción de Miguel Morey. Barcelona: Paidós, 2005.

DELEUZE, G.; GUATTARI, F. Mil mesetas. Capitalismo y esquizofrenia. Traducción de José Vázquez Pérez. Valencia: Pre-textos, 2002.

DERRIDA, J. Forcenar al subjetil. Traducción de Bruno Mazzoldi. Bogotá: Pontificia Universidad Javeriana, 2011.

DESCARTES, R. Meditaciones metafísicas (1641). Traducción de Guillermo Graíño Ferrer. Madrid: Alianza Editorial, 2011.

DUMOULIÉ, C. Nietzsche et Artaud. Pour une éthique de la cruauté. Paris: PUF, 1992.

NIETZSCHE, F. La ciencia jovial. "La gaya scienza”. Traducción de José Jara. Caracas: Monte Avila Editores, 1990.

NIETZSCHE, F. El origen de la tragedia. Traducción de Eduardo Ovejero Mauri. Madrid: Espasa Calpe, 2007.

NIETZSCHE, F. Fragmentos póstumos (1885-1889). Volumen IV. Traducción de Juan Luis Vermal y Joan B. Llinares. Madrid: Tecnos, 2008.

NIETZSCHE, F. Fragmentos póstumos (1875-1882). Volumen II. Traducción de Manuel Barrios y Jaime Aspiunza. Madrid: Tecnos, 2008b.

NIETZSCHE, F. Fragmentos póstumos (1881-1885). Volumen III. Traducción de Diego Sánchez Meca y Jesús Conill. Madrid: Tecnos, 2010.

STIEGLER, B. Nietzsche e la biologia. Traducción de Federico Leoni. Mantova: Negretto editore, 2010.

VERNANT, J. P. Mito y pensamiento en la Grecia antigua. Traducción de Juan Diego López Bonillo. Barcelona: Ariel, 1973. 